\title{
Genomic evidence of paternal genome elimination in globular springtails
}

Kamil S. Jaron ${ }^{1,{ }^{*}}$, Christina N. Hodson ${ }^{1}$, Jacintha Ellers ${ }^{2}$, Stuart JE Baird ${ }^{3}$, Laura Ross ${ }^{1}$

$5{ }^{1}$ Institute of Evolutionary Biology, University of Edinburgh, Edinburgh, EH9 3JT, UK ${ }^{2}$ Department of Ecological Science, Vrije Universiteit Amsterdam, Faculty of Science, Amsterdam, $1081 \mathrm{HV}, \mathrm{NL}$ ${ }^{3}$ Institute of Vertebrate Biology, Czech Academy of Sciences, Květná 8, Brno, 60365, CZ

*Corresponding author: kamiljaron@gmail.com

\section{Abstract}

Paternal genome elimination (PGE) - a type of reproduction in which males inherit but fail to pass on their father's genome - evolved independently in six to eight arthropod clades.

Thousands of species, including several important for agriculture, reproduce via this mode of reproduction. While some of the clades are well established PGE systems, the evidence in globular springtails (Symphypleona) remains elusive, even though they represent the oldest and most species rich clade putatively reproducing via PGE. We sequenced genomic DNA from whole bodies of Allacma fusca males with sufficiently high fractions (31 - 38\%) of sperm to conclusively confirm that all the sperm carry one parental haplotype only. Although it is suggestive that the single haplotype present in sperm is maternally inherited, definitive genetic proof of the parent of origin is still needed. The genomic approach we developed allows for detection of genotypic differences between germline and soma in all species with sufficiently high fraction of germline in their bodies. This opens new opportunities for scans for reproductive modes in small animals.

\section{Introduction}

The mechanism of reproduction varies considerably across the tree of life (Bachtrog et al., 2014; Normark, 2003). Historically, cytological comparisons of male and female karyotypes have been used to determine the mode of reproduction in a species. However, cytological studies are labour intensive and not all species have visible sex-specific

30 karyotypes. As a consequence, many species still have undefined reproductive systems. On the other hand, genomic techniques have been successfully deployed to identify sex chromosomes in many taxa such as Diptera (Anderson et al., 2020; Vicoso \& Bachtrog, 2015), and Lepidoptera (Fraïsse et al., 2017) and more recently to understand the exact form of parthenogenesis in species such as californian stick insects (Jaron et al., 2021), and bdelloid rotifers (Simion et al., 2021). Now, it is time to consider the ways we can use genomic techniques to study other modes of reproduction such as paternal genome elimination.

Paternal genome elimination is a reproduction system in which males develop from fertilised eggs, but pass to the next generation only the maternally inherited haplotype (see (Burt \& Trivers, 2006) for a clear introduction to the topic). The inheritance pattern is exactly the same as in better known haplodiploidy (arrhenotoky), in which males develop from 
unfertilized haploid eggs, but mechanistically they represent very different reproductive systems. Similar to haplodiploidy, there are only a few known transitions to PGE (six to eight), and PGE clades are frequently very old and diverse. Thousands of arthropod species reproduce via some form of paternal genome elimination including numerous agricultural pests (scale insects, Hessian fly, lucerne flea) and even pest control species (phytoseiid mites). However, the occurrence of PGE is likely significantly under-reported as it can be hard to confirm. It tends to occur in small arthropods that are poorly studied and hard to 50 culture under laboratory conditions, making it challenging to study inheritance patterns. For example, PGE was only demonstrated in Liposcelis lice and human head and body lice (order Psocodea) very recently through genetic crosses tracking alleles over several generations, (de la Filia et al., 2018; Hodson et al., 2017) even though meiosis was known to be unusual in lice for decades prior to this (Cannon, 1922; Doncaster \& Cannon, 1920).

55 Because of the difficulty of inheritance studies, many of the reported cases are based on indirect evidence, usually cytogenetic observations of unusual chromosome behaviour (Supplementary Table 1).

Part of the reason PGE is difficult to identify, is that individual clades differ greatly in the 60 mechanism of PGE, and hence require different types of evidence for confirmation (Figure 1). In all PGE species males develop from fertilized diploid eggs, and always exclusively transmit maternally inherited chromosomes to offspring. However, they differ in the processes leading to the elimination of paternal chromosomes. For example, in Phytoseiidae mites and some armored scale insects, the paternal genome is completely eliminated early

65 in embryogenesis in a process called embryonic PGE (Brown, 1965; Nelson-Rees et al., 1980). The fact that males are completely haploid soon after fertilisation makes this type of PGE easy to detect in genetic and cytological studies, although it can be hard to distinguish from true haplodiploidy. The two can be distinguished, however, via carefully designed phenotypic or irradiation crosses (Helle et al., 1980; Hoy, 1979), by cytology of early embryogenesis (Nelson-Rees et al., 1980), or by observing whether unfertilised eggs develop into males (Häußermann et al., 2020). 
bioRxiv preprint doi: https://doi.org/10.1101/2021.11.12.468426; this version posted November 14,2021. The copyright holder for this preprint (which was not certified by peer review) is the author/funder, who has granted bioRxiv a license to display the preprint in perpetuity. It is made available under aCC-BY 4.0 International license.

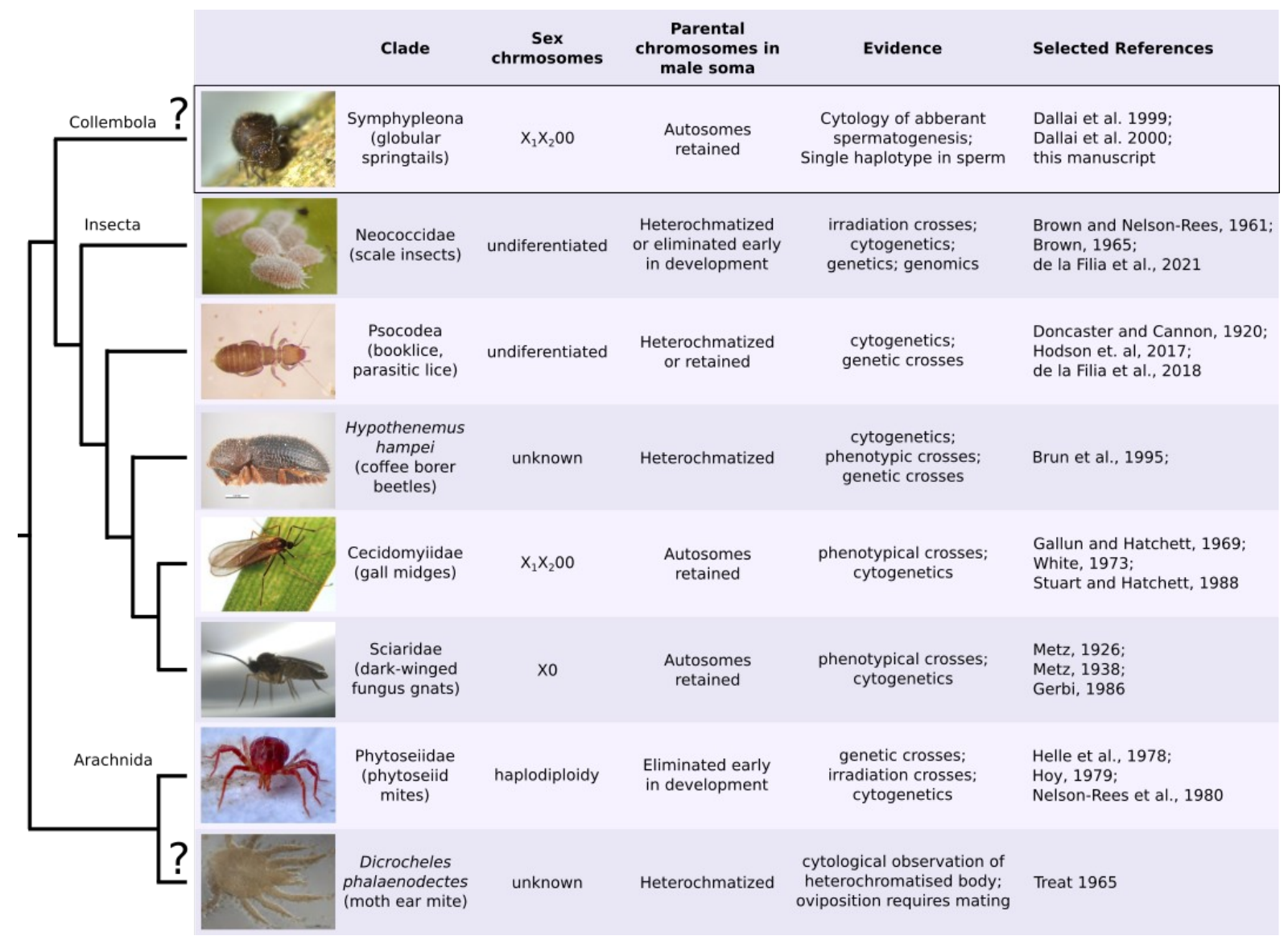

Figure 1. Clades with suggested paternal genome elimination (PGE) and evidence

75 supporting it. The cladogram shows the phylogenetic relationships between putative (marked by "?") and confirmed PGE clades. Although all PGE clades exhibit transmission dynamics where paternally inherited chromosomes are not transmitted to offspring through males, the sex chromosome system and the treatment/expression of paternally inherited chromosomes in male somatic cells can differ between and within clades. A more detailed

80 list of relevant literature is in SM Table 1. Two taxa without definitive proofs of PGE are marked "?". Image credits: mealybugs (scale insects) by Andrew J. Mongue, coffee borer beetles by Walker, K., phytoseiid mite by Mick Talbot.

85 In other types of PGE, males fully or partially retain their paternal genome throughout development and paternal chromosomes are excluded during spermatogenesis only, hence these types are known as germline PGE. While paternal chromosomes are retained, they form a dense heterochromatic body at the periphery of the cell nuclei for most scale insects (Brown, 1965; Ross et al., 2012), the coffee borer beetle (Brun et al., 1995), booklice

90 (Hodson et al., 2017), and potentially in some Leapideae mites (Treat, 1965). This distinctive feature is not a formal test of PGE, but allows potential PGE species to be easily detected using cytological observation. It also means that males in these clades are mostly haploid in terms of gene expression, despite their diploid karyotype (Brun et al., 1995; de la Filia et al., 2021). A combination of embryonic and spermatogenic elimination is found in two dipteran

95 families: fungus gnats, and gall midges. Males of these clades exclude one or two paternal chromosomes in early embryogenesis (usually referred to as X chromosomes), while retaining all other chromosomes in their soma. The remaining paternal chromosomes are lost during aberrant spermatogenesis. In fungus gnats and gall midges it has been shown by 
bioRxiv preprint doi: https://doi.org/10.1101/2021.11.12.468426; this version posted November 14, 2021. The copyright holder for this preprint (which was not certified by peer review) is the author/funder, who has granted bioRxiv a license to display the preprint in perpetuity. It is made available under aCC-BY 4.0 International license.

crosses that all the eliminated chromosomes in both embryogenesis and spermatogenesis are of paternal origin (Gallun \& Hatchett, 1969; Metz, 1926, 1938; Stuart \& Hatchett, 1988). Finally a similar type of PGE has been suggested to occur in globular springtails. However the evidence is solely based on unusual chromosome behaviour and no inheritance studies are available.

105 Globular springtails are a large and species-rich order with enormous importance for soil ecology (Hopkin, 1997). Their karyotype consists of four to five autosomes and two sex chromosomes reffered as $X_{1}$ and $X_{2}$ (Dallai et al., 2000, 2004). As in PGE flies, male globular springtail zygotes are initially fully diploid, but during very early embryogenesis males eliminate one copy of the $X_{1}$ and $X_{2}$ chromosomes (Supplementary figure 1) (Dallai et al., 110 2000). Furthermore, the aberrant spermatogenesis also shows a great similarity to the two dipteran families. During meiosis I of spermatogenesis the two $X$ chromosomes cosegregate, hence half of the secondary spermatocytes carry all six chromosomes and the other half contain the four autosomes only (Figure 2). The $X$ chromosome lacking spermatocytes immediately degenerate, and only the spermatocytes with the complete

115 chromosome set undergo a second meiotic division to form two haploid spermatids (Dallai et al., 2000). In a series of papers, Dallai and colleagues described this type of aberrant spermatogenesis in five globular springtail families, namely Dicyrtomidae (Dallai et al., 1999), Sminthuridae (Dallai et al., 2000), Bourletiellidae (Dallai et al., 2001), Sminthurididae and Katiannidae (Dallai et al., 2004). This is likely the ancestral state of the Symphypleona

120 order. Hence, it is clear that one full haploid set of chromosomes gets eliminated during development (X chromosomes) and spermatogenesis (autosomes) of males. However, it remains unclear whether the chromosome elimination is random during meiosis or systematically dependent on the parental origin (i.e. PGE).

125 There is no distinct name for the putative sex chromosome constelation in globular springtails. It is best described as PGE X0, although the absence of $X$ chromosomes in males is not the primary sex determination. Other springtail orders in contrast have regular meiosis (Dallai et al., 1999) and X0 or XY sex determination (Hemmer, 1990; Núñez, 1962).

130 We investigated possible approaches to confirm PGE in globular springtails. First, we considered conducting genetic crosses of Allacma fusca, a relatively common and large globular springtail commonly found in woodland areas across Europe. However, wild-caught globular springtails are hard to maintain in lab conditions. Alternatively, genotyping a male and its sperm can at least inform us if all sperm contain a single haplotype only, presumably the maternal one. While investigating methods to efficiently sequence male sperm, we discovered male bodies contain a large fraction of sperm (up to $38 \%$ of cells) and sequencing whole bodies seems to be the most efficient way to sequence sperm, although it requires in-silico bioinformatics analysis to separate the effect of somatic and germline genomes in the sequencing library. With our innovative approach we demonstrated that the

140 set of autosomes co-segregate with the $X_{1}$ and $X_{2}$ chromosomes, implying uniparental inheritance. 


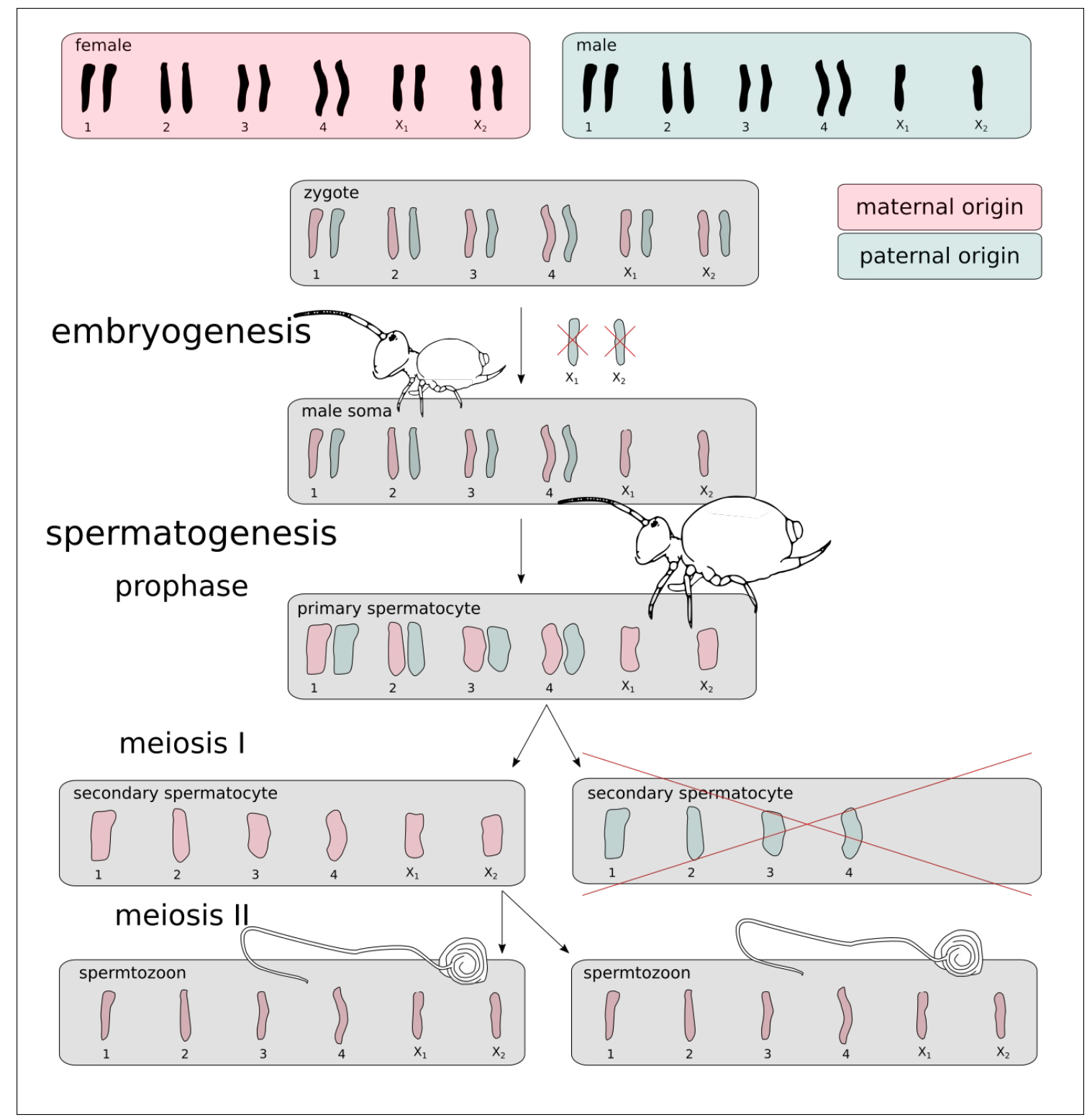

Figure 2: Scheme of PGE in globular springtails. Male springtail zygotes are initially diploid for all chromosomes. One copy of chromosomes $X_{1}$ and $X_{2}$ is excluded during early embryogenesis. Adult males then generate a half of their secondary spermatocytes with the remaining $X$ chromosomes, and a half without $X_{1}$ and $X_{2}$ that degenerates immediately. The scheme and cartoonized shapes of chromosomes are based on (Dallai et al., 2000). Note the spermatozoon "tail" is not flagellum, as flagellum is densely coiled, see (Dallai et al., 2009) for details. The coloring is assuming reproduction via paternal genome elimination as tested in this study. 


\section{Materials and Methods}

\section{Springtails collected and sequenced}

We used an assembly (GCA_910591605.1) and sequencing reads (sample accession

ERS6488033) we previously generated for a male Allacma fusca individual (Anderson et al., 2020). We also collected 12 additional $A$. fusca samples for resequencing. The sex of individual samples was determined from the modality of sequencing coverage and revealed 11 of 12 resequenced samples were females (SM Figure 1). The resequenced male individual was sampled at Blackford Hill (sample id BH3-2, ERS6377982), Edinburgh, Scotland (55.924039, -3.196509). We isolated the DNA using Qiagen DNeasy Blood and tissue kit extraction protocol and sequenced using the Illumina HiSeq platform. The standard adapters and low quality bases were trimmed using skewer v๑.2.2 with options $-m$ pe $-n-q$ 26 - 121 (Jiang et al., 2014). We used both the male and all female libraries to identify Xlinked scaffolds. Although the reference genome is fragmented, reliable chromosomal assignments are available for $170.6 \mathrm{Mbp}$, representing $40.1 \%$ of the assembly span. In total, 77.9 Mbp of scaffolds are X-linked, while 92.7 Mbp are autosomal (SM Text 1).

All analyses were also performed on the genome of an outgroup species Orchesella cincta (GCA_001718145.1, (Faddeeva-Vakhrusheva et al., 2016)). Both male O. cinta resequencing data (ERS7711323) and chromosomal assignments were taken from (Anderson et al., 2020). Orchesella cincta is a distantly related springtail with X0 sex determination (Hemmer, 1990) and therefore ideal as a negative control for this study.

\section{Calculation of k-mer coverages}

All male sequencing libraries were initially subjected to quality control using kmer spectra analysis. We calculated the k-mer coverage histogram with $\mathrm{k}=21$ using KMC3 (Kokot et al., 2017) and visualized it using GenomeScope 2.0 (Ranallo-Benavidez et al., 2020).

GenomeScope fits a model estimating error rate, genome size and heterozygosity assuming all chromosomes are of the same ploidy. This assumption is clearly violated in male globular springtails, hence we disregarded the genome size and heterozygosity estimates for these samples and used the software only for characterising 1 ) the monoploid (1n) k-mer coverage representing the two $X$ chromosomes and the autosomal heterozygous loci, 2 ) the diploid (2n) k-mer coverage representing homozygous autosomal regions and 3 ) the error peak indicating overall quality of the sequencing run.

In sequencing libraries of a tissue with AAXO karyotype, the autosomes are expected to have exactly twice the coverage of $X$ chromosomes (i.e. the library has evenly spaced peaks). However, k-mer coverages displayed unevenly spaced $1 \mathrm{n}$ and $2 \mathrm{n}$ peaks for the reference $A$. fusca male (SM Figure 2A), the BH3-2 A. fusca had insufficient coverage to confirm the pattern (SM Figure 2C), but the $O$. cincta male showed evenly spaced $1 \mathrm{n}$ and $2 \mathrm{n}$ peaks (SM Figure 2E). To clarify the signal and replicate the pattern we also estimated mapping coverage (see Box 1 for the difference between the two). 
bioRxiv preprint doi: https://doi.org/10.1101/2021.11.12.468426; this version posted November 14, 2021. The copyright holder for this preprint (which was not certified by peer review) is the author/funder, who has granted bioRxiv a license to display the preprint in perpetuity. It is made available under aCC-BY 4.0 International license.

\section{Calculation of mapping coverages}

We mapped trimmed sequencing libraries to the softmasked reference genomes of Allacma fusca (GCA_910591605.1) and Orchesella cincta respectively (GCA_001718145.1). The reads were mapped using bowtie2 with the parameter - -very-sensitive-local (Langmead \& Salzberg, 2012). We used samtools depth to extract per base coverage (Li et al., 2009), and calculated per scaffold mean coverage. Then we estimated $1 \mathrm{n}$ and $2 \mathrm{n}$ coverage peaks using kernel smoothing with kernel width chosen by Sheather and Jones method (bw = "SJ") (Sheather \& Jones, 1991) while weighted by scaffold length (weights = scf_tab\$len / sum(scf_tab\$len)).

This method resulted in both $A$. fusca males showing distinctively uneven peaks (SM Figure 2B and $\mathbf{D}$ ), while the $O$. cincta male again showed the expected $1 \mathrm{n}$ and $2 \mathrm{n}$ coverage peaks. These findings prompted us to create a two tissue model that can explain the shift of relative positions of the two peaks.

\section{Box 1: Different types of sequencing coverages used in this manuscript}

Sequencing coverage is the mean number of times every position in a genome is represented in reads. Sequencing coverage is usually estimated by dividing the total sequencing yield by the haploid genome size. However, in many cases, for genome analyses of non-model organisms the level of contamination in sequencing libraries; sequencing errors; and genome size is unknown. This can make estimating sequencing coverage challenging. Hence there are multiple other ways to measure and estimate sequencing coverage. These different measures have different properties and are used for different purposes. K-mer coverage is the mean number of occurrences of each unique continuous genomic sequence of length $\mathrm{k}$ in reads. K-mer decomposition is independent of a reference genome and the coverage is estimated by fitting a model to $\mathrm{k}$-mer coverage histogram (Ranallo-Benavidez et al., 2020). This reference-free technique is well suited to observing raw signals from data unbiased by complicated procedures such as genome assembly. Mapping coverage is the mean number of reads mapping to each position of a haploid reference genome. It is dependent on the quality of the reference and quality of mapping. The main advantage is that reads of heterozygous sites as well as infrequent sequencing errors still typically map to the same position on the reference. Hence, this coverage is suited the best to estimate ploidy of each reference genomic region. Allelic coverage is also derived from sequencing reads mapped to the genome. However, the mapped reads usually have PCR duplicates marked and are subsequently used for calling variants. The coverage is then the number of non-duplicated reads supporting individual alleles. Sequencing errors do not contribute to this coverage. For the sake of completeness, in theory, k-mer coverage $\left(C_{k}\right)$ is convertible to mapping coverage $\left(C_{m}\right)$ by a simple approximation $C_{k} \approx C_{m} \frac{R-k+1}{R}$

Where $k$ is the length of $\mathrm{k}-\mathrm{mer}, R$ is the length of reads. However, in practice there are usually too many issues - no haploid reference is perfect and the mapping process is also dependent on many assumptions. Hence in practice the two measures need to be calculated independently. 


\section{Two tissue model}

250 The unevenly spaced mapping coverage (see Box 1 ) peaks of $X$ chromosomes and autosomes implies the sequencing library contained tissues with various ploidies. A simple model that can explain the pattern is a two tissue mixture model - a mixture of a tissue with $1: 2 X$ to autosome ratio (e.g. male soma) and a tissue with $1: 1 \times$ to autosome ratio (e.g. secondary spermatocytes or sperms). Using the X-chromosome and autosome mapping coverage peaks, we can estimate the relative contribution of the two tissue types to the sequencing library (Supplementary figure 5A) and the fraction of the two tissues. Assuming the $1: 1$ tissue is haploid, the relative fraction of that tissue $\left(f_{h}\right)$ in the sequencing library is $f_{h}=1-\frac{\left(c_{A}-c_{X}\right)}{c_{X}}$ where $C_{A}$ is the mapping coverage of the autosomal peak, and $C_{X}$ is the mapping coverage of the $\mathrm{X}$ chromosome peak.

The only described tissue with 1:1 $\mathrm{X}$ to autosome ratio in adult male globular springtails are primary and secondary spermatocytes, spermatids, and spermatozoon (Dallai et al., 2000). Hence, it is probably safe to assume this is the tissue that is causing the relative mapping coverage shift illustrated in Supplementary figure 5B (for alternative unsupported hypotheses tested to explain the 1n mapping coverage shift, see SM Text 2).

\section{Testing PGE}

270 Sequencing a mixture of sperm and soma provides us a chance to test previously suggested paternal genome elimination (PGE) in globular springtails. The PGE inheritance model (Figure 2) predicts that the sperm contain only the maternally inherited haploid set of chromosomes $\left(\mathrm{A}_{\mathrm{m}} \mathrm{X}_{\mathrm{m}}\right)$. As all the autosomes present in the haploid sperm are of maternal origin, all the heterozygous autosomal loci should display a higher coverage support of

275 maternal alleles compared to paternal. In the ideal case, we would like to compare coverage support of phased haplotypes, which is unfeasible with fragmented reference genomes and short read libraries. Instead, we separated the alleles of heterozygous autosomal variants to the "major" and "minor" alleles - representing the variants with higher and lower coverage support respectively. Under the PGE model the maternal and paternal alleles are expected

280 to have vastly different coverage support, therefore the "major" alleles will be vastly of maternal origin, while the "minor" alleles will be vastly paternal. The fraction of possible misassigned variants was explored through modeling of sequencing coverages using negative binomial distributions with parameters estimated from expected sequencing coverages (Figure 4).

Furthermore, under the PGE model, the distribution of maternal allele coverage depths is expected to resemble the distribution of $\mathrm{X}$-chromosome allele coverage depths. Due to a small fraction of misassigned alleles in males (as explained in the previous paragraph) the match is not expected to be exactly perfect. The expected levels of imperfect match were also estimated via the same set of simulated coverages.

We performed the same analysis on the genome of male O. cincta and two A. fusca 
females. The two females only show the decomposition of autosomal heterozygous alleles in the case of frequent misassignment of maternal and paternal alleles (as they are generated from the same coverage distribution, SM Figure 3). The $O$. cinca male further allows the same comparison of decomposed allele coverages to the distribution of coverage of alleles found on the $X$ chromosome.

Before calling variants we marked duplicates in the mapping files using picard

300 MarkDuplicates (Picard Toolkit, 2019) and called variants using freebayes v1.3.2-dirty (Garrison \& Marth, 2012) with stringent input base and mapping quality filters as well as required minimal allele coverage (--standard-filters --min-coverage 5 ), but we relaxed the priors of Hardy-Weinberg proportions are it might not be met in a PGE population (- hwe-priors-off), while assuming diploidy (- $\left.\begin{array}{ll}-p & 2\end{array}\right)$. The raw variant calls were subsequently filtered for high quality variants ( $f$ "QUAL $>20$ ") only using vcffilter from the vcflib library version 1.0.0_rc3 (Garrison et al., 2021) and sorted to autosomal and X-linked using a custom python script. The variants sorted to chromosomes were plotted using $\mathrm{R}$ scripts.

\section{Independent estimate of the relative fraction of the haploid tissue}

The described test for PGE requires moderate to high levels of heterozygosity to provide enough power to estimate the coverage distributions of the putatively maternal and paternal alleles. Low heterozygosity in combination with stringent parameters for SNP calling could cause low statistical power to test PGE via called variants.

To avoid the lack of power and any other potential biases introduced via SNP calling, we deployed an alternative approach as a supplementary analysis. For the two Almaca males we counted sequence states aligned under the GCA_910591605.1 reference using samtools mpileup converted to matrix form with Popoolation2 mpileup2sync (Kofler et al., 2011). After

320 filtering out scaffolds with evidence of copy-number variation between the males (SM Figure 4), we calculated minor frequencies $p_{p}$ for all genomic positions with at least two states in the pileup. Then we examine the distribution of variant sites by minor allele frequency for both males. See SM Text 4 for details.

325 As all the sperm is expected to contain only maternally inherited autosomes, the expected proportion $p_{p}$ of paternal (green shaded in Figure 2) automosomes over all the body's cells is $\frac{1-f_{h}}{2-f_{h}}$. The expected allele coverage ratio (site frequency) of the paternal state is $p_{p}$, this is the minority state when $f_{h}>0$, and $f_{h}=\frac{1-2 p_{p}}{1-p_{p}}$

330 which allows us to estimate the relative fraction of haploid tissue from the estimated allele coverage ratios. We also present this analysis for heterozygous SNP calls, allowing comparison of the two methods.

All scripts and materials are available online at https://github.com/RossLab/genomic- 


\section{Results}

The analysis of trimmed sequencing libraries of the two $A$. fusca male individuals revealed that both have unexpected relative $\mathrm{k}$-mer and mapping coverages of the $\mathrm{X}$ chromosomes compared to the autosomes (see Box 1 for coverage definitions). In both cases the $1 \mathrm{n}$ coverage estimates were more than half of the diploid coverage estimate. We estimated the $\mathrm{X}$-chromosome and autosomal mapping coverages in the $\mathrm{BH} 3-2$ male to be $18.35 \mathrm{x}$ and 29.65x respectively (Figure 3 ), deviating from the 1:2 ratio, under which we would expect the diploid coverage to be $36.7 x$ (or haploid coverage of $14.8 x$ ). A remarkably similar uneven spacing of the $X$ and autosomal peaks was observed in the reference $A$. fusca male for both k-mer and mapping coverages (57.7x and 95.2x), while in a strong contrast with a male sequencing library of a non-PGE species $O$. cincta, where the two coverage peaks were nearly perfectly spaced (Supplementary figure 2).

Using the coverage estimates and the two tissues mixture model (see Methods and $35 \%$ (the reference male) and 38\% (BH3-2). Furthermore, for the $\mathrm{BH} 3-2$ individual we estimated the expected allele coverages for paternal (11.3x) and maternal (18.35x) autosomes and X chromosomes (18.35x) (Figure 3).

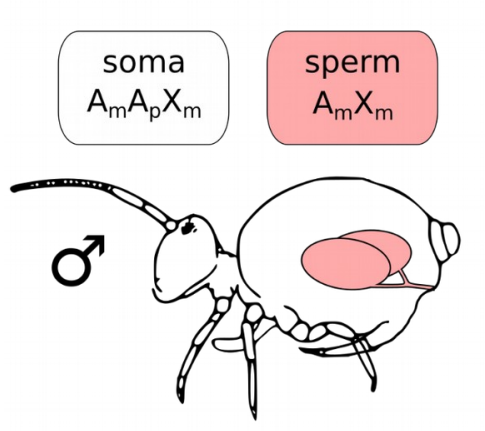

\begin{tabular}{|c|c|c|}
\hline chr & tissue & exp. coverage \\
\hline $\mathrm{X}_{\mathrm{m}}$ & $\Xi$ & $18.35 \mathrm{x}$ \\
\hline $\mathrm{X}_{\mathrm{p}}$ & - & - \\
\hline $\mathrm{A}_{\mathrm{m}}$ & $\Xi$ & $18.35 \mathrm{x}$ \\
\hline $\mathrm{A}_{\mathrm{p}}$ & $\square$ & $11.3 \mathrm{x}$ \\
\hline
\end{tabular}

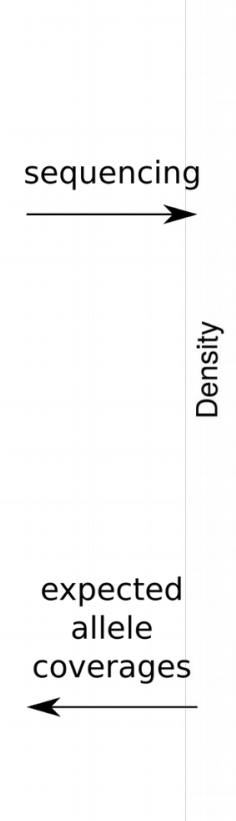

355

Figure 3: Overview of expected coverages potentially explaining the shift of the coverage peaks. PGE is expected to cause the shift of coverage peaks due to a significant proportion of sperm in the body, as indicated in cartoons (explained in greater detail in Supplementary figure 5). The table contains the expected paternal and maternal coverages of autosomes and X-chromosomes for the male resequencing individual $\mathrm{BH} 3-2$.

The expected coverages of maternal and paternal autosomes and $X$ chromosomes were compared with the distribution of allelic coverages of variants on autosomes and $X$ chromosomes. After quality filtering we identified 28,070 and 235,301 heterozygous variants anchored to chromosomes in the reference and BH3-2 individuals respectively (SM Table 
bioRxiv preprint doi: https://doi.org/10.1101/2021.11.12.468426; this version posted November 14, 2021. The copyright holder for this preprint (which was not certified by peer review) is the author/funder, who has granted bioRxiv a license to display the preprint in perpetuity. It is made available under aCC-BY 4.0 International license.

2). The extremely low heterozygosity of the reference male reduces the power to use the sample for testing the PGE hypothesis and is discussed in SM Text 3. Of the BH3-2 anchored heterozygous variants 227,570 were located on autosomal scaffolds, while only 7,731 heterozygous variants on X-linked scaffolds, indicating low levels of false positives among variant calls (less than 100 false positives per 1Mbp). On the other hand, we identified 60,999 homozygous variant calls on the $X$ linked scaffolds that were used for the comparison with allele coverages of the autosomal variants. The coverage supports of $A$. fusca male were contrasted to 1,959,258 heterozygous autosomal variants and 400,001 homozygous $X$-linked variants in the outgroup species $O$. cincta (non-PGE springtail).

We decomposed the male heterozygous autosomal variants in both samples to the "major" and "minor" alleles - representing the variants with higher and lower coverage support respectively. The mean coverage of maternal variants $(18.35 \mathrm{x})$ is expected to be higher compared to the coverage of paternal variants (11.3x), hence although it is possible some of the paternal variants will be by chance higher, this will affect only a very small fraction of the variants. To quantify the effect of misassigning variants by coverage, we simulated the coverage support of maternal and paternal alleles under the PGE model using the negative binomial distribution (Figure 4A). The simulation with 200000 variants showed that the probability of missassigment is 0.162 (Binom. $\mathrm{Cl}$ : $0.1608748,0.1641082)$. On the other

385 hand, applying the same decomposition of heterozygous variants to "major" and "minor" in non-PGE species leads to $\sim 0.5$ of misassigned variants (by definition) (Figure 4B). In both cases the black distribution in the background represents the background distribution for the maternal variants. In the real data, we used the homozygous variants located on the $X$ chromosomes to estimate the coverage distribution of monoploid maternal alleles. Under the

390 PGE model, we expect it to roughly overlap with the "major" variant coverage peak (Figure $4 \mathrm{~A}$ ), contrasting to the non-PGE model where the expected distribution will be exactly in the middle of the "major" and "minor" coverage peaks (Figure 4B).

We confirm the coverage supports of "major" and "minor" autosomal variants in A. fusca 395 male BH3-2 are close to the expected coverages (Figure 4C) generated from the two tissue mixture model (Figure 3). The fit is not perfect, probably due to problems with misassigned alleles. Furthermore, the distribution of "major" autosomal variants closely resembles the distribution of homozygous X-linked variants, with similar levels of disagreement compared to the simulated data (Figure 4A). Both comparisons together provide a strong support for

400 the PGE model in Allacma fusca. The analysis of $A$. fusca shows a clearly different pattern to $O$. cincta, a springtail with standard spermatogenesis. The decomposed coverage supports display largely overlapping distributions and the coverage distribution of $\mathrm{X}$-linked variants is nearly located intermediate between the peaks of "major" and "minor" allele coverages (Figure 4D), as predicted by the non-PGE model (Figure 4B). Note that the first coverage 405 peak of $X$-linked variants displays spurious and unexpected coverages, which according to the genome profiling (SM Figure 2E) should be considered false positives. 

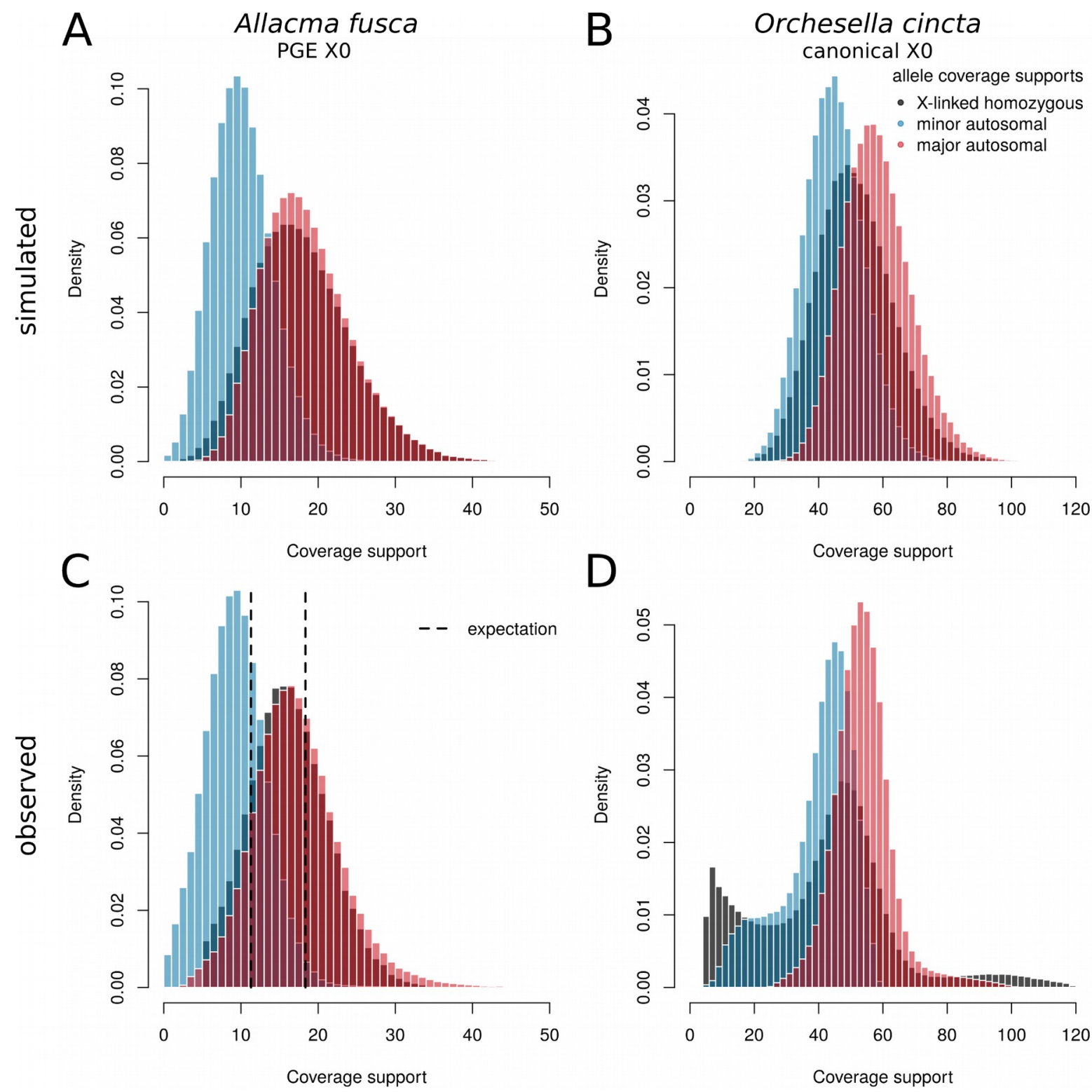

D

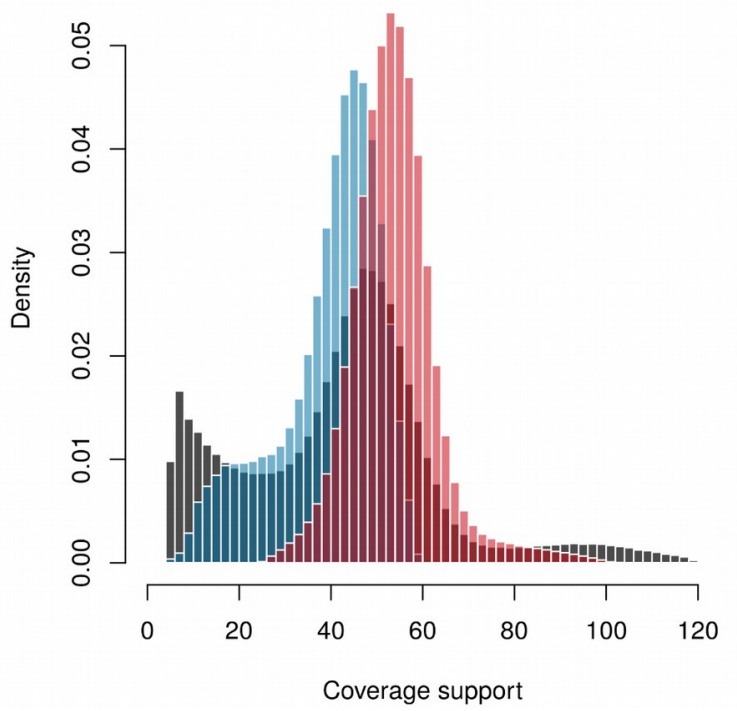

Figure 4: Decomposed heterozygous allele coverage supports. Coverage supports of

410 the two alleles of heterozygous sites are decomposed to those with higher coverage ("major", in red) and lower coverage ("minor", in blue). These are compared to coverage supports of homozygous $X$-linked variants. Panels $\mathbf{A}$ and $\mathbf{B}$ are simulated allele coverages for a PGE X0 system and non-PGE X0 system. In PGE species (A), major alleles vastly represent maternal alleles and show similar coverage distributions to

415 homozygous X-linked alleles (maternal haploid). In canonical X0 system (B) the decomposition also leads to bimodal distribution, however, the $\mathrm{X}$-linked allele has an intermediate coverage peak in between of the two autosomal distributions. The observed coverage distributions in Allacma fusca (C) strongly support the PGE model. The major allele coverage support distribution closely reassembles the distribution of homozygous

$420 \mathrm{X}$-linked alleles as well as matching the expected coverage calculated from the $1 \mathrm{n}$ coverage shift (Figure 3). In contrast Orchesella cincta (D), a species with regular spermatogenesis and $\mathrm{XO}$ sex determination, shows patterns consistent with the expected properties of canonical sexual XO sex determination species with $\mathrm{X}$-linked coverage support intermediate of the decomposed autosomal coverage supports. 
bioRxiv preprint doi: https://doi.org/10.1101/2021.11.12.468426; this version posted November 14, 2021. The copyright holder for this preprint (which was not certified by peer review) is the author/funder, who has granted bioRxiv a license to display the preprint in perpetuity. It is made available under aCC-BY 4.0 International license.

We utilised an analysis of raw pileups to create an independent estimate of the fraction of the haploid tissue $f_{h}$ from the estimated minor allele frequency of all genomic positions with two states located on scaffolds with no signs of copy number variation (See SM Text 4 and SM Figure 4). This approach showed a higher abundance of bistates around coverage ratios 0.397 in Afus1 and 0.406 in BH3-2 (SM Figure 5), indicating that even the reference male shows some detectable heterozygous states, but with much noisier signal compared to $\mathrm{BH} 3-2$. The estimated fraction of sperm in the bodies from the paternal allele frequency $p_{p}$ are $33.96 \%$ for Afus1 and $31.39 \%$ for $\mathrm{BH} 3-2$ individuals respectively.

\section{Discussion}

We estimated that a large proportion of a male adult $A$. fusca body (31 - 38\%) consists of secondary spermatocytes, spermatids or mature sperm (from now on collectively referred to as sperm). Although the estimated fraction is relatively high, it is in agreement with high production of spermatophores by Allacma fusca (Dallai et al., 2009) and the estimate does not surpass that of other invertebrates. Caenorhabditis elegans can carry around 2000 germ cells, while their soma consist of precisely 959 cells (Sulston \& Horvitz, 1977). Germ cells therefore represent $\sim 67 \%$ of $C$. elegans cell count. A similar case is found among arthropods: Up to $75 \%$ of body cells in Daphnia males are sperm (Dufresne et al., 2019). It is important to note we specifically discuss the fraction of cells, not the biomass, as presumably, sperm are substantially smaller compared to other cell types in the body.

Taking advantage of the high sperm fraction, we demonstrated that all the sperm have exactly the same genotype which conclusively implies co-segregation of full chromosomal sets under the absence of recombination in this globular springtail. This conclusion was also supported by analyses of read pileups (SM Text 4 and SM Figure 5). The analyses of pileups further revealed that the reference sample indeed also shows uneven coverage ratios of heterozygous alleles, although this signal was much weaker compared to the $\mathrm{BH} 3-2$ individual. We propose the reference individual could have reduced heterozygosity due to local inbreeding of the population that was sampled. Altogether, all results are in agreement with the PGE model (Figure 2) that has been previously proposed (Dallai et al., 2000).

We have shown a set of chromosomes is eliminated, but not whether the eliminated set is maternal or paternal. To provide definitive proof of PGE we would have to genotype both parents of a male as well as its sperm. Hypothetically, the eliminated chromosomes could be maternal. However, the elimination of maternal chromosomes during spermatogenesis has only even been observed in a rare form of androgenesis (Schwander \& Oldroyd, 2016), a reproductive system in which males fertilize a female of a closely related sexual strain and cause elimination of the maternal genome as found in freshwater clam Corbicula leana (Komaru et al., 1998) or Australian carp gudgeons (Majtánová et al., 2021). However, this form of androgenesis requires a co-existence of lineages with canonical sexual reproduction with male androgenetic lineages, which is extremely unlikely in the case of globular springtails as the aberrant spermatogenesis seems to be present already in the common ancestor of globular springtails (Dallai et al., 1999, 2000, 2001, 2004). Paternal genome elimination on the other hand is a mode of reproduction that is conserved in at least six large 
bioRxiv preprint doi: https://doi.org/10.1101/2021.11.12.468426; this version posted November 14, 2021. The copyright holder for this preprint (which was not certified by peer review) is the author/funder, who has granted bioRxiv a license to display the preprint in perpetuity. It is made available under aCC-BY 4.0 International license.

470 clades (Figure 1) and although with our data we also cannot completely exclude the possibility that the non-random chromosome elimination is associated with a different, as yet undescribed, evolutionary phenomena, paternal genome elimination is the only explanation compatible with known biology.

475 In particular, globular springtail reproduction most closely resembles the reproductive cycle of two dipteran families that also eliminate paternal chromosomes both in early spermatogenesis (what we call $X$ chromosomes in these species) and during spermatogenesis (Gerbi, 1986; Metz, 1938). In both these two families females are frequently monogenic - each female produce broods of single sex only (Metz, 1931). So far 480 this has not been tested in globular springtails, probably because they are both difficult to cultivate and show very little sexual dimorphism. Finally, the third genomic peculiarity found in both PGE fly families - they carry germ-line restricted chromosomes (Hodson et al., 2021; Metz, 1938), is a feature that is not shared in globular springtails as no differences between germ-line and soma karyotypes have been reported other than the aberrant

485 spermatogenesis discussed in detail above.

Although we have tested this hypothesis in only a single globular springtail species Allacma fusca, the same type of aberrant spermatogenesis was demonstrated in seven species of five different families (Supplementary table 1) (Dallai et al., 1999, 2000, 2001, 2004). The

490 most parsimonious explanation of the aberrant spermatogenesis in all the examined species is that PGE is the ancestral feature of globular springtails. Although we expect most of the globular springtails to retain this type of reproduction, there are multiple transitions to parthenogenetic reproduction (reviewed in (Chernova et al., 2010)). Other PGE clades usually show high conservation of this reproduction mode (Brown, 1965; Gerbi, 1986; Ross

495 et al., 2012), the only known exception is found in louse. The human body louse seems to show a partial reversal to non-PGE sexual type of reproduction (de la Filia et al., 2018; McMeniman \& Barker, 2005). Whether or not any globular springtail species have reverted to a more canonical type of reproduction is however an open question for further research.

500 Our study strongly suggests that globular springtails are the oldest and most species-rich clade reproducing via PGE. With 15,600 species estimated worldwide (Porco et al., 2014) globular springtails are a great clade to study the long term consequences of coping with PGE over hundreds of millions of years of evolution (Leo et al., 2019). This unusual mode of inheritance is likely to profoundly influence their evolutionary history. Recent theory suggests

505 that haplodiploidy and PGE affect the evolution of reproductive isolation and increase diversification rates (Lohse \& Ross, 2015; Patten et al., 2015). Springtails provide a great opportunity to test this theory as three of four springtail orders are species rich and allow us to estimate rates of diversification.

510 Paternal genome elimination also affects the dynamics of sexual conflict as shown in recently developed models (Hitchcock et al., 2021; Klein et al., 2021). Notably, it changes the relative role of $X$ chromosomes and autosomes. Under PGE both $X$ chromosomes and autosomes show bias in transmission between generations and sex alternation (see (Klein et al., 2021) for details), however, $X$ chromosomes in globular springtails are also subjected to

515 haploid selection in males. Unlike in species with normal diploid reproduction, the dominance of male beneficial alleles is the only factor that determines if they are more likely to get fixed on $\mathrm{X}$ chromosome (for recessive alleles) or anywhere in the genome (for dominant alleles) 
(Klein et al., 2021). Therefore comparing the levels of sexual antagonism on $X$ chromosomes and autosomes in globular springtails will allow the effect of dominance in sexual selection to be quantified, which has been a central question of sex chromosome evolution.

Besides rich biological interpretation, our study demonstrates the power of a careful bioinformatics analysis of whole genome sequencing data. The initial observation of suspiciously spaced coverage peaks was discovered during a routine quality control step using k-mer spectra analysis. Systematic scans for mixtures of two tissues in a single library could be more widely utilised to detect any peculiarities in reproduction that cause differences of karyotypes of male germline and soma such as the peculiar type of PGE in globular springtails.

\section{Acknowledgements}

First of all, we would like to thank Tanja Schwander for the invaluable suggestion of sperm being the cause of disrupted coverage ratio of autosomes and $X$ chromosomes in males. We would also like to thank Andrew Mongue and the rest of Ross lab members for comments on early versions of this manuscript. Wytham Woods, City of Edinburgh Council, and Friends of the Hermitage of Braid for permission to collect springtails. $\mathrm{CH}$ would like to thank NSERC and the Darwin Trust of Edinburgh for postgraduate financial support. LR would like to acknowledge funding from the European Research Council Starting Grant (PGErepo) and from the Dorothy Hodgkin Fellowship DHFIR1|180120. Financial support from $\mathrm{NIH/GM121455}$ to SAG is gratefully acknowledged.

\section{References}

Anderson, N., Jaron, K. S., Hodson, C. N., Couger, M. B., Ševčík, J., Pirro, S., Ross, L., \& Roy, S. W. (2020). Gene-rich X chromosomes implicate intragenomic conflict in the evolution of bizarre genetic systems. BioRxiv, 2020.10.04.325340. https://doi.org/10/ gjtw99

Bachtrog, D., Mank, J. E., Peichel, C. L., Kirkpatrick, M., Otto, S. P., Ashman, T.-L., Hahn, M. W., Kitano, J., Mayrose, I., Ming, R., Perrin, N., Ross, L., Valenzuela, N., Vamosi, J. C., \& Tree of Sex Consortium. (2014). Sex determination: Why so many ways of doing it? PLoS Biology, 12(7), e1001899. https://doi.org/10/gfkh5h

Brown, S. W. (1965). Chromosomal survey of the armored and palm scale insects (Coccoidea: Diaspididae and Phoenicococcidae). Hilgardia, 36, 189-294.

Brun, L. O., Stuart, J., Gaudichon, V., Aronstein, K., \& French-Constant, R. H. (1995). 
Functional haplodiploidy: A mechanism for the spread of insecticide resistance in an Important international insect pest. Proceedings Of The National Academy Of

Sciences Of The United States Of America, 92(21), 9861-9865.

https://doi.org/10/b39wtm

Burt, A., \& Trivers, R. L. (2006). Genes in Conflict. Harvard University Press.

Cannon, H. G. (1922). Memoirs: A further Account of the Spermatogenesis of Lice. Journal of Cell Science, s2-66(264), 657-667. https://doi.org/10/gm6t8m

Chernova, N. M., Potapov, M. B., Savenkova, Yu. Yu., \& Bokova, A. I. (2010). Ecological significance of parthenogenesis in collembola. Entomological Review, 90(1), 23-38. https://doi.org/10/c97kts

Dallai, R., Fanciulli, P. P., Carapelli, A., \& Frati, F. (2001). Aberrant spermatogenesis and sex determination in Bourletiellidae (Hexapoda, Collembola), and their evolutionary significance. Zoomorphology, 120(4), 237-245. https://doi.org/10/c7fr66

Dallai, R., Fanciulli, P. P., \& Frati, F. (1999). Chromosome elimination and sex determination in springtails (Insecta, Collembola). Journal of Experimental Zoology, 285(3), 215225. https://doi.org/10/cq83zc

570 Dallai, R., Fanciulli, P. P., \& Frati, F. (2000). Aberrant spermatogenesis and the peculiar mechanism of sex determination in symphypleonan Collembola. Journal of Heredity, 91(5), 351-358. https://doi.org/10/dkgjmt

Dallai, R., Fanciulli, P. P., \& Frati, F. (2004). New data on the aberrant spermatogenesis of Collembola. Pedobiologia, 48(5-6), 487-492. https://doi.org/10/bs7rmg

575 Dallai, R., Zizzari, Z. V., \& Fanciulli, P. P. (2009). Different sperm number in the spermatophores of Orchesella villosa (Geoffroy) (Entomobryidae) and Allacma fusca (L.) (Sminthuridae). Arthropod Structure \& Development, 38(3), 227-234. https://doi.org/10/fk6gvz

de la Filia, A. G., Andrewes, S., Clark, J. M., \& Ross, L. (2018). The unusual reproductive system of head and body lice (Pediculus humanus). Medical and Veterinary Entomology, 32(2), 226-234. https://doi.org/10/gf22ks 
de la Filia, A. G., Mongue, A. J., Dorrens, J., Lemon, H., Laetsch, D. R., \& Ross, L. (2021). Males That Silence Their Father's Genes: Genomic Imprinting of a Complete Haploid Genome. Molecular Biology and Evolution, 38(6), 2566-2581. https://doi.org/10/gm74zf

Doncaster, L., \& Cannon, H. G. (1920). Memoirs: On the Spermatogenesis of the Louse (Pediculus Corporis and P. Capitis), With Some Observations on the Maturation of the Egg. Journal of Cell Science, s2-64(255), 303-325. https://doi.org/10/gm6t8k

Dufresne, F., Belzile, C., McKindsey, C., \& Beaudreau, N. (2019). Sperm number assessed by flow cytometry in species of Daphnia (Crustacea, Cladocera). Invertebrate Biology, 138(3), e12261. https://doi.org/10/gf759v

Faddeeva-Vakhrusheva, A., Derks, M. F. L., Anvar, S. Y., Agamennone, V., Suring, W., Smit, S., van Straalen, N. M., \& Roelofs, D. (2016). Gene Family Evolution Reflects Adaptation to Soil Environmental Stressors in the Genome of the Collembolan Orchesella cincta. Genome Biology and Evolution, 8(7), 2106-2117. https://doi.org/10/f8zg59

Fraïsse, C., Picard, M. A. L., \& Vicoso, B. (2017). The deep conservation of the Lepidoptera Z chromosome suggests a non-canonical origin of the W. Nature Communications, 8(1), 1486. https://doi.org/10/gcmbnr

600 Gallun, R. L., \& Hatchett, J. H. (1969). Genetic Evidence of Elimination of Chromosomes in the Hessian Fly1,2. Annals of the Entomological Society of America, 62(5), 10951101. https://doi.org/10/gm74zd

Garrison, E., Kronenberg, Z. N., Dawson, E. T., Pedersen, B. S., \& Prins, P. (2021). Vcflib and tools for processing the VCF variant call format (p. 2021.05.21.445151). https://doi.org/10.1101/2021.05.21.445151

Garrison, E., \& Marth, G. (2012). Haplotype-based variant detection from short-read sequencing. ArXiv:1207.3907 [q-Bio]. http://arxiv.org/abs/1207.3907

Gerbi, S. A. (1986). Unusual Chromosome Movements in Sciarid Flies. In W. Hennig (Ed.), Germ Line-Soma Differentiation (pp. 71-104). Springer. 
Häußermann, C. K., Giacobino, A., Munz, R., Ziegelmann, B., Palacio, M. A., \& Rosenkranz, P. (2020). Reproductive parameters of female Varroa destructor and the impact of mating in worker brood of Apis mellifera. Apidologie, 51(3), 342-355. https://doi.org/10/gmw43n parthenogenesis in the false spider mites (Acari: Tenuipalpidae). Genetica, 54(1), 45-50. https://doi.org/10/brsjf9

Hemmer, W. (1990). Karyotype differentiation and chromosomal variability in springtails (Collembola, Insecta). Biology and Fertility of Soils, 9(2), 119-125. https://doi.org/10/ dkqp9j

Hitchcock, T. J., Gardner, A., \& Ross, L. (2021). Sexual antagonism in haplodiploids. BioRxiv, 2021.03.26.437233. https://doi.org/10/gm8ds2

Hodson, C. N., Hamilton, P. T., Dilworth, D., Nelson, C. J., Curtis, C. I., \& Perlman, S. J. (2017). Paternal Genome Elimination in Liposcelis Booklice (Insecta: Psocodea). Genetics, 206(2), 1091-1100. https://doi.org/10/gfzgjf

Hodson, C. N., Jaron, K. S., Gerbi, S., \& Ross, L. (2021). Evolution of gene-rich germline restricted chromosomes in black-winged fungus gnats through introgression (Diptera: Sciaridae). BioRxiv, 2021.02.08.430288. https://doi.org/10/gmhhw4 Hopkin, S. P. (1997). Biology of Springtails (Insecta: Collembola). Oxford University Press. 630 Hoy, M. A. (1979). Parahaploidy of the "Arrhenotokous" Predator, Metaseiulus Occidentalis (acarina: Phytoseiidae) Demonstrated by X-Irradiation of Males. Entomologia Experimentalis et Applicata, 26(1), 97-104. https://doi.org/10/cmjzk7

Jaron, K. S., Parker, D. J., Anselmetti, Y., Van, P. T., Bast, J., Dumas, Z., Figuet, E., François, C. M., Hayward, K., Rossier, V., Simion, P., Robinson-Rechavi, M., Galtier, N., \& Schwander, T. (2021). Convergent consequences of parthenogenesis on stick insect genomes (p. 2020.11.20.391540). https://doi.org/10.1101/2020.11.20.391540 Jiang, H., Lei, R., Ding, S.-W., \& Zhu, S. (2014). Skewer: A fast and accurate adapter 
trimmer for next-generation sequencing paired-end reads. BMC Bioinformatics, 15(1), 182. https://doi.org/10/gb8wj4

640 Klein, K., Kokko, H., \& ten Brink, H. (2021). Disentangling Verbal Arguments: Intralocus Sexual Conflict in Haplodiploids. The American Naturalist, 000-000. https://doi.org/10/gm8dsz

Kofler, R., Pandey, R. V., \& Schlötterer, C. (2011). PoPoolation2: Identifying differentiation between populations using sequencing of pooled DNA samples (Pool-Seq). Bioinformatics, 27(24), 3435-3436. https://doi.org/10/cjmkn5

Kokot, M., Długosz, M., \& Deorowicz, S. (2017). KMC 3: Counting and manipulating k-mer statistics. Bioinformatics, 33(17), 2759-2761. https://doi.org/10/f96gjp

Komaru, A., Kawagishi, T., \& Konishi, K. (1998). Cytological evidence of spontaneous androgenesis in the freshwater clam Corbicula leana Prime. Development Genes and Evolution, 208(1), 46-50. https://doi.org/10/dz4n4n

Langmead, B., \& Salzberg, S. L. (2012). Fast gapped-read alignment with Bowtie 2. Nature Methods, 9(4), 357-359. https://doi.org/10/gd2xzn

Leo, C., Carapelli, A., Cicconardi, F., Frati, F., \& Nardi, F. (2019). Mitochondrial Genome Diversity in Collembola: Phylogeny, Dating and Gene Order. Diversity, 11(9), 169. https://doi.org/10/ggcdpd

Li, H., Handsaker, B., Wysoker, A., Fennell, T., Ruan, J., Homer, N., Marth, G., Abecasis, G., Durbin, R., \& 1000 Genome Project Data Processing Subgroup. (2009). The Sequence Alignment/Map format and SAMtools. Bioinformatics, 25(16), 2078-2079. https://doi.org/10/ff6426

660 Lohse, K., \& Ross, L. (2015). What haplodiploids can teach us about hybridization and speciation. Molecular Ecology, 24(20), 5075-5077. https://doi.org/10/f7wbwv Majtánová, Z., Dedukh, D., Choleva, L., Adams, M., Ráb, P., Unmack, P. J., \& Ezaz, T. (2021). Uniparental Genome Elimination in Australian Carp Gudgeons. Genome Biology and Evolution, 13(6). https://doi.org/10/gnbjts

665 McMeniman, C. J., \& Barker, S. C. (2005). Transmission ratio distortion in the human body 
louse, Pediculus humanus (Insecta: Phthiraptera). Heredity, 96(1), 63-68.

https://doi.org/10/cd936g

Metz, C. W. (1926). Genetic Evidence of a Selective Segregation of Chromosomes in Sciara (Diptera). Proceedings of the National Academy of Sciences, 12(12), 690-692. https://doi.org/10/d97phw

Metz, C. W. (1931). Unisexual Progenies and Sex Determination in Sciara. The Quarterly Review of Biology, 6(3), 306-312. https://doi.org/10/dzfwck

Metz, C. W. (1938). Chromosome behavior, inheritance and sex determination in Sciara. American Naturalist, 72, 485-520. https://doi.org/10/d9nw39

675 Nelson-Rees, W. A., Hoy, M. A., \& Roush, R. T. (1980). Heterochromatinization, chromatin elimination and haploidization in the parahaploid mite Metaseiulus occidentalis (Nesbitt) (Acarina: Phytoseiidae). Chromosoma, 77(3), 263-276. https://doi.org/10/cspk6x

Normark, B. B. (2003). The evolution of alternative genetic systems in insects. Annual Review of Entomology, 48, 397-423. https://doi.org/10/bggbqz

Núñez, O. (1962). Cytology of Collembola. Nature, 194(4832), 946-947. https://doi.org/10/bwpxxc

Patten, M. M., Carioscia, S. A., \& Linnen, C. R. (2015). Biased introgression of mitochondrial and nuclear genes: A comparison of diploid and haplodiploid systems. Molecular Ecology, 24(20), 5200-5210. https://doi.org/10/gf4ftd

Picard toolkit. (2019). Broad Institute, GitHub repository: http://broadinstitute.github.io/picard/. http://broadinstitute.github.io/picard/

Porco, D., Skarżyński, D., Decaëns, T., Hebert, P. D. N., \& Deharveng, L. (2014). Barcoding the Collembola of Churchill: A molecular taxonomic reassessment of species diversity in a sub-Arctic area. Molecular Ecology Resources, 14(2), 249-261. https:// doi.org/10/f5svff

Ranallo-Benavidez, T. R., Jaron, K. S., \& Schatz, M. C. (2020). GenomeScope 2.0 and Smudgeplot for reference-free profiling of polyploid genomes. Nature 
Communications, 11(1), 1432. https://doi.org/10/ggrbdk

695 Ross, L., Shuker, D. M., Normark, B. B., \& Pen, I. (2012). The role of endosymbionts in the evolution of haploid-male genetic systems in scale insects (Coccoidea). Ecology and Evolution, 2(5), 1071-1081. https://doi.org/10/f4jdfb

Schwander, T., \& Oldroyd, B. P. (2016). Androgenesis: Where males hijack eggs to clone themselves. Philosophical Transactions of the Royal Society of London B: Biological Sciences, 371(1706), 20150534. https://doi.org/10/gfxd92

Sheather, S. J., \& Jones, M. C. (1991). A Reliable Data-Based Bandwidth Selection Method for Kernel Density Estimation. Journal of the Royal Statistical Society: Series B (Methodological), 53(3), 683-690. https://doi.org/10/gfttr8

Simion, P., Narayan, J., Houtain, A., Derzelle, A., Baudry, L., Nicolas, E., Arora, R., Cariou, M., Cruaud, C., Gaudray, F. R., Gilbert, C., Guiglielmoni, N., Hespeels, B., Kozlowski, D. K. L., Labadie, K., Limasset, A., Llirós, M., Marbouty, M., Terwagne, M., ... Van Doninck, K. (2021). Chromosome-level genome assembly reveals homologous chromosomes and recombination in asexual rotifer Adineta vaga. Science Advances, 7(41), eabg4216. https://doi.org/10.1126/sciadv.abg4216

710 Stuart, J. J., \& Hatchett, J. H. (1988). Cytogenetics of the Hessian Fly: II. Inheritance and Behavior of Somatic and Germ-Line-Limited Chromosomes. Journal of Heredity, 79(3), 190-199. https://doi.org/10/gm74zb

Sulston, J. E., \& Horvitz, H. R. (1977). Post-embryonic cell lineages of the nematode, Caenorhabditis elegans. Developmental Biology, 56(1), 110-156. https://doi.org/10/b48mn4

Treat, A. E. (1965). Sex-Distinctive Chromatin and the Frequency of Males in the Moth Ear Mite. Journal of the New York Entomological Society, 73(1), 12-18.

Vicoso, B., \& Bachtrog, D. (2015). Numerous Transitions of Sex Chromosomes in Diptera. PLoS Biology, 13(4), e1002078. https://doi.org/10/f7crnn 